\title{
XI. Electromagnets.-II. On the magnetic permeability of iron and steel, with a new theory of magnetism
}

\section{R.H.M. Bosanquet}

To cite this article: R.H.M. Bosanquet (1885) XI. Electromagnets.-II. On the magnetic permeability of iron and steel, with a new theory of magnetism , Philosophical Magazine Series 5, 19:117, 73-94, DOI: $10.1080 / 14786448508627650$

To link to this article: http://dx.doi.org/10.1080/14786448508627650

曲 Published online: 29 Apr 2009.

Submit your article to this journal $[\pi$

Џll Article views: 2

Q View related articles $\sqsubset$ 
LONDON, EDINBURGH, AND DUBLIN

\title{
PHILOSOPHICAL MAGAZINE
}

\author{
AND \\ JOURNAL OF SCIENCE.
}

[FIFTH SERIES.]

FEBRUARY 1885 .

XI. Electromagnets.-II. On the Magnetic Permeability of Iron and Steel, with a new Theory of Magnetism. By $\mathrm{R} . \mathrm{H}$. M. Bosanquet, St. John's College, Oxford.

To the Editors of the Philosophical Magazine and Journal. Gentuemen,

REFORE attempting to draw rigorous experimental conclusions as to the effect of the magnetic properties of Iron and Steel on electromagnets made of these metals, it is necessary to obtain some further information as to the magnetic properties in question.

Hitherto I have accepted the results of Rowland's experiments with rings*; but I have now repeated and varied these experiments, with special regard to the following points:-

To find the average properties assigned by this method to the Iron and Steel I commonly use;

To assign to the results formulæ of a more general and more manageable type than that employed by Rowland;

And to justify experimentally the assumption that the permeability, as calculated from the experiments, is independent of the size and proportions of the rings examined.

A new theory of these magnetic properties will be enunciated and applied.

The iron upon which most of the following experiments have been made is what is called ordinary bar-iron. It is what I commonly use for electromagnets. It is moderately soft and admits of being easily forged. It is stamped with a

$$
\text { * Phil. Mag. [4] xlvi. p. } 140 \text { (1873). }
$$

Phil. Mag. S. 5. Vol. 19. No. 117. Feb. 1885. 
crown, and I shall speak of it as crown iron. One ring of best Lowmoor has also been examined.

The steel ring $\mathbf{J}$ was forged from the remainder of the bar of cast steel from which the divided magnet was made, which is called A in my paper I. on Permanent Magnets (Phil. Mag. August 1884, p. 142). It was first examined soft, and then after hardening.

The method employed was substantially that of Rowland. The rings were uniformly wound with coils through which the magnetizing current was transmitted. The current employed was a small derived current from the circuit of the dynamo machine; so that it could be opened, closed, or reversed without upsetting the main circuit. The current was measured by means of two galvanometers of Helmholtz's pattern, the one having two coils, the other eighteen. The galvanometers were always erected on the brick stand which forms the standard position for which values of $\mathrm{H}$ are determined in the laboratory.

The observations were all made by reversal.

A number of induction-coils, varying from 250 to 1 , were wound round the ring to be examined. In circuit with these coils were the ballistic galvanometer and the earth inductioncoil, for the reduction of the readings to absolute measure.

Two earth induction-coils were employed. The first had 21 turns, with a mean diameter of 51.30 centim.; the other 250 turns, with a mean circumference of 166.53 centim. With both of these coils the impulse of the galvanometer due to half a turn about a vertical axis in the standard position* was about $7^{\circ}$. But in the case of the larger coil, a deflection corresponds to about twelve times as great an induction as with the smaller coil, so that a very large range is secured. The galvanometer has a circular scale which reads, by reflection, directly to $5^{\prime}$ and by estimation to single minutes.

The mode of calculation adopted is precisely equivalent to that of Rowland. Rowland's M is current-turns per unit length, $=\frac{\mathrm{C} n}{l}$ in our notation. Then his expression connecting $\mu$, the permeability, with the total number of lines of force is,

$$
\text { lines of force }=4 \pi \frac{\mathrm{C} n}{l} \mu\left(\pi \mathrm{R}^{2}\right) \text {; }
$$

and for the magnetic induction, or lines of force per unit area,

* The positions of rest between which the half turn is made are such that the plane of the coil is at right angles to the magnetic meridian. Hence the coil cuts the lines of force of $\mathrm{H}$ twice in the half turn. 
we have

$$
\mathfrak{B}=4 \pi \frac{\mathrm{Cn}}{l} \mu .
$$

Now $4 \pi \mathrm{C} n$ is the total magnetic potential exerted on the ring by the magnetizing coil ; whence

$$
\frac{\text { potential }}{\mathfrak{B} \mathbf{B}}=\frac{l}{\mu},
$$

which is what I call the magnetic resistance*. $\mu$ is then calculated really both by Rowland and myself from the formula

$$
\mu=\frac{l \mathbf{B}}{\text { potential }}=\frac{l}{\text { magnetic resistance }} ;
$$

though of course the form and the letters Rowland uses are different.

The whole process of calculation of an experiment is embodied in the following formulæ.

Let $\alpha$ be the deflection of the ballistic galvanometer due to reversal, with $m$ induction-coils ; then

$$
2 m \mathrm{SAB}=\kappa \propto
$$

( $\mathbf{S}=$ sectional area of bar $)$.

Let $\beta=$ deflection due to half turn of earth induction-coil ( $\mathrm{N}=$ number of coils, $A=$ mean area); then

$$
2 \mathrm{NAH}=\kappa \beta \text {. }
$$

Combining these, we have

$$
\mathbf{B}=\frac{\alpha}{\beta} \frac{\mathrm{NAH}}{m \mathrm{~S}} \text {. }
$$

Let $G$ be that part of the tangent-galvanometer coefficient which is independent of $H$, so that we have for the current

Then potential

$$
\mathrm{C}=\mathrm{GH} \tan \delta \text {. }
$$

magnetic resistance

$$
=4 \pi n \mathrm{C}=4 \pi n \mathrm{GH} \tan \delta \text {, }
$$

$$
\rho=\frac{\text { potential }}{\mathbf{B}},
$$

which is independent of $\mathrm{H}$; and

$$
\mu=\frac{l}{\rho}
$$

where $l$ is the mean circumference of the ring.

The following Tables exhibit the results of the various

* See Phil. Mag. xvii. p. 531. 
experiments thus made. They also show the degree of accuracy attained by the Fourier's series, by which I have endeavoured to represent $\mu$ as a function of $\mathbf{2 B}$.

E. Forged Ring Crown Iron.

Mean diameter . . $d=10.035$ centim.

Bar-thickness . . $2 \mathrm{R}=1.298$ "

Number of coils $. n=\left\{\begin{array}{l}462 \\ 486\end{array}\right.$

$\mu$ calc. $=144+221 \cos \theta$

where

$+2000\left\{\sin \theta+\frac{1}{6} \sin 2 \theta+\frac{1}{18} \sin 3 \theta+\frac{3}{40} \sin 4 \theta\right\}$,

$$
\theta=\frac{\mathfrak{B}}{100} \text { degrees. }
$$

\begin{tabular}{|c|c|c|c|c|c|c|c|}
\hline \multirow{2}{*}{ BB. } & \multicolumn{2}{|c|}{$\mu}$. & \multirow{2}{*}{ Diffs. } & \multirow{2}{*}{ 3B. } & \multicolumn{2}{|c|}{$\mu}$. & \multirow{2}{*}{ Diffs } \\
\hline & Obs. & Cale. & & & Obs. & Calc. & \\
\hline $129 \cdot 7$ & 437 & 437 & 0 & 11516 & 1615 & 1625 & +10 \\
\hline $239 \cdot 1$ & 493 & 498 & +5 & 13276 & 1381 & 1207 & -174 \\
\hline $2174 \cdot 3$ & 1474 & 1470 & -4 & 14017 & 1047 & 1017 & -30 \\
\hline 3186.9 & 1817 & 1836 & +19 & 15000 & 635 & 732 & $\begin{array}{r}+97 \\
\end{array}$ \\
\hline $5181 \cdot 7$ & 2163 & 2199 & +36 & 16380 & 218 & 349 & +131 \\
\hline $6403 \cdot 4$ & 2234 & 2229 & -5 & 17245 & 117 & 119 & $\begin{array}{r}2 \\
+\quad 2\end{array}$ \\
\hline $7208 \cdot 4$ & 2206 & 2197 & -9 & & & & \\
\hline
\end{tabular}

F. Forged Ring Crown Iron.

Mean diameter . . $d=22 \cdot 1$ centim.

Bar-thickness . . $2 \mathrm{R}=1 \cdot 292$ "

Number of coils. . $n=1118$.

$\mu$ calc. $=200+1630\left\{\sin \theta+\frac{1}{4} \sin 2 \theta+\frac{1}{9} \sin 3 \theta+\frac{1}{16} \sin 4 \theta\right\}$, where

$$
\theta=\frac{\mathbf{a}}{100} \text { degrees. }
$$

\begin{tabular}{|c|c|c|c|c|c|c|c|}
\hline \multirow{2}{*}{ B. } & \multicolumn{2}{|c|}{$\mu$. } & \multirow{2}{*}{ Diffs. } & \multirow{2}{*}{36.} & \multicolumn{2}{|c|}{$\mu}$. & \multirow{2}{*}{ Diffs. } \\
\hline & Obs. & Calc. & & & Obs. & Calc. & \\
\hline $15 \cdot 108$ & 208 & 208 & 0 & $6438 \cdot 4$ & 1825 & 1847 & +22 \\
\hline $142 \cdot 55$ & 360 & 284 & -76 & $7655 \cdot 3$ & 1704 & 1749 & $\begin{array}{r}+45 \\
\end{array}$ \\
\hline 512.97 & 587 & 502 & -85 & 10767 & 1581 & 1481 & -100 \\
\hline $2783 \cdot 8$ & 1592 & 1572 & -20 & 12537 & 1252 & 1258 & +6 \\
\hline $3616 \cdot 5$ & 1784 & 1784 & & 13900 & 1000 & 989 & -11 \\
\hline $4282 \cdot 6$ & 1869 & 1858 & -11 & 15025 & 692 & 749 & +57 \\
\hline $5746 \cdot 6$ & 1895 & 1890 & -5 & 18834 & 150 & 60 & -90 \\
\hline
\end{tabular}




\section{G. Forged Ring Crown Iron.}

Mean diameter . . $d=21.5$ centim.

Bar-thickness . . $2 \mathrm{R}=2.535 \quad$,

Number of coils $\quad n=989$.

$\mu$ calc. $=290\left(1+\frac{1}{2} \cos \theta\right)+2000\left\{\sin \theta+\frac{1}{4} \sin 2 \theta\right\}$, where

$$
\theta=\frac{\mathbf{B}}{100} \text { degrees. }
$$

\begin{tabular}{|c|c|c|c|c|c|c|c|}
\hline \multirow{2}{*}{ Z3. } & \multicolumn{2}{|c|}{$\mu}$. & \multirow{2}{*}{ Diffs. } & \multirow{2}{*}{ ZB. } & \multicolumn{2}{|c|}{$\mu}$. & \multirow{2}{*}{ Diffs. } \\
\hline & Obs. & Calc. & & & Obs. & Calo. & \\
\hline $50 \cdot 61$ & $46 \mathrm{I}$ & 461 & 0 & 11970 & 1555 & 1525 & -30 \\
\hline $448 \cdot 17$ & 765 & 669 & -96 & 13710 & 1192 & 1047 & -45 \\
\hline $1972 \cdot 5$ & 1615 & 1419 & -196 & 14426 & 832 & 867 & +35 \\
\hline 63637 & 2501 & 2544 & +43 & 15505 & 664 & 620 & -44 \\
\hline $9003 \cdot 3$ & 2241 & 2289 & +48 & 16042 & 395 & 512 & +117 \\
\hline 11090 & 1763 & 1773 & +10 & 17536 & 145 & 230 & +85 \\
\hline
\end{tabular}

H. Forged Ring Crown Iron.

Mean diameter . . $d=10 \cdot 735$ centim.

Bar-thickness . . 2R= $\cdot 7137$ "

Number of coils . $n=560$.

where

$\mu$ calc. $=217+164 \cos \theta+1400\left\{\sin \theta+\frac{1}{4} \sin 2 \theta\right\}$,

$$
\theta=\frac{\mathfrak{B}}{100} \text { degrees. }
$$

\begin{tabular}{|c|c|c|c|c|c|c|c|}
\hline \multirow{2}{*}{ 37. } & \multicolumn{2}{|c|}{$\mu$} & \multirow{2}{*}{ Diffs. } & \multirow{2}{*}{ 3. } & \multicolumn{2}{|c|}{$\mu}$. & \multirow{2}{*}{ Diffs. } \\
\hline & Obs. & Calc. & & & Obs. & Calc. & \\
\hline $34 \cdot 375$ & 395 & 394 & -1 & 11261 & 1134 & 1198 & +64 \\
\hline 286.85 & 418 & 486 & +68 & 13355 & 885 & 769 & -116 \\
\hline $1079 \cdot 4$ & 817 & 769 & -48 & 14501 & 630 & 557 & -73 \\
\hline $6025 \cdot 5$ & 1797 & 1816 & +19 & 14718 & 502 & 519 & +17 \\
\hline $7704 \cdot 6$ & 1717 & 1771 & +54 & 15409 & 254 & 407 & +153 \\
\hline 84357 & 1710 & 1681 & -29 & 17642 & 97 & 98 & +1 \\
\hline $9739 \cdot 6$ & 1354 & 1498 & +144 & & & & \\
\hline
\end{tabular}


K. Forged Ring Crown Iron.

Mean diameter . . $d=22.725$ centim.

Bar-thickness . . 2R $=.7544$,

Number of coils . $n=1217$.

where

$\mu$ calc. $=380 \cos \theta-30+1770\left\{\sin \theta+\frac{1}{4} \sin 2 \theta_{\}}\right.$,

$$
\theta=\frac{\mathfrak{B}}{100} \text { degrees. }
$$

\begin{tabular}{|c|c|c|c|c|c|c|c|}
\hline \multirow{2}{*}{ \$B. } & \multicolumn{2}{|c|}{$\mu$. } & \multirow{2}{*}{ Diffs. } & \multirow{2}{*}{ 3B. } & \multicolumn{2}{|c|}{$\mu$} & \multirow{2}{*}{ Diffs. } \\
\hline & Obs. & Calc. & & & Obs. & Oalc. & \\
\hline $67 \cdot 293$ & 422 & 381 & -41 & $9598 \cdot 2$ & 1557 & 1660 & +103 \\
\hline 293.43 & 428 & 486 & +58 & 10413 & 1531 & 1384 & -147 \\
\hline $2174 \cdot 7$ & 1288 & 1278 & -10 & 11511 & 1104 & 1357 & +253 \\
\hline $2337 \cdot 5$ & 1176 & 1343 & +167 & 12148 & 799 & 953 & +154 \\
\hline 3949.0 & 1766 & 1823 & $\begin{array}{r}+57 \\
+57\end{array}$ & 13246 & 666 & 569 & -97 \\
\hline $4710 \cdot 5$ & 1982 & 1967 & -15 & 13104 & 505 & 618 & +113 \\
\hline $5719 \cdot 9$ & 2070 & 2067 & -3 & 13671 & 358 & 466 & +108 \\
\hline $8677 \cdot 4$ & 1914 & 1808 & -106 & 15053 & 135 & 131 & -4 \\
\hline $8889 \cdot 8$ & 1775 & 1764 & -11 & & & & \\
\hline
\end{tabular}

I. Forged Ring Best Lowmoor Iron.

Mean diameter . . $d=10.025$ centim.

Bar-thickness . . 2R=1.293 "

Number of coils $\quad n=\left\{\begin{array}{l}470 \\ 476\end{array}\right.$

$\mu$ calc. $=250+1800\left\{\sin \theta+\frac{1}{4} \sin 2 \theta+\frac{1}{9} \sin 3 \theta+\frac{1}{16} \sin 4 \theta\right\}$, where

$$
\theta=\frac{\mathfrak{B}}{100} \text { degrees. }
$$

\begin{tabular}{|c|c|c|c|c|c|c|c|}
\hline \multirow{2}{*}{ (3. } & \multicolumn{2}{|c|}{$\mu$. } & \multirow{2}{*}{ Diffs. } & \multirow{2}{*}{ 3B. } & \multicolumn{2}{|c|}{$\mu}$. & \multirow{2}{*}{ Diffs. } \\
\hline & Obs. & Calc. & & & Obs. & Calc. & \\
\hline 32.944 & 271 & 272 & +1 & $7090 \cdot 9$ & 2173 & 2012 & -161 \\
\hline $429 \cdot 15$ & 617 & 547 & -70 & 8193.9 & 1935 & 1915 & -20 \\
\hline 1321.8 & 1024 & 1078 & +54 & $9690 \cdot 8$ & 1842 & 1795 & -47 \\
\hline $1623 \cdot 6$ & 1427 & 1247 & -180 & 10400 & 1483 & 1730 & +247 \\
\hline $2395 \cdot 1$ & 1558 & 1616 & $\begin{array}{r}+58 \\
\end{array}$ & 13159 & 1188 & 1390 & +202 \\
\hline 4541.5 & 2072 & 2116 & $\begin{array}{r}1 \\
+44\end{array}$ & 15050 & 818 & 851 & +33 \\
\hline $6324 \cdot 2$ & 2080 & 2077 & $\begin{array}{r}1 \\
-\quad 3\end{array}$ & 16309 & 592 & 57 & -18 \\
\hline $6712 \cdot 3$ & 1993 & 2045 & +52 & 16939 & 444 & 448 & +4 \\
\hline $6700 \cdot 1$ & 2033 & 2046 & +13 & 19303 & 155 & 182 & +37 \\
\hline
\end{tabular}


J. Forged Ring Cast Steel (Soft).

Mean diameter . . $d=14.25$ centim.

Bar-thickness . . 2R=1.421 ,

Number of coils . . $n=1074$.

where

$$
\mu \text { calc. }=120+340 \sin \theta \text {, }
$$

$$
\theta=\frac{\mathfrak{B}}{100} \text { degre日s. }
$$

\begin{tabular}{|c|c|c|c|c|c|c|c|}
\hline \multirow{2}{*}{38.} & \multicolumn{2}{|c|}{$\mu}$. & \multirow{2}{*}{ Diffs. } & \multirow{2}{*}{38.} & \multicolumn{2}{|c|}{$\mu$. } & \multirow{2}{*}{ Diffs. } \\
\hline & Obs. & Calc. & & & Obs. & Oalc. & \\
\hline $21 \cdot 873$ & 126 & 121 & -5 & 5815.9 & 420 & 409 & -11 \\
\hline $83 \cdot 360$ & 120 & 125 & +5 & 9467.8 & 461 & 459 & $-\quad 2$ \\
\hline $277 \cdot 3$ & 156 & 136 & -20 & 11497 & 443 & 428 & -15 \\
\hline 1825.9 & 248 & 226 & -22 & 12443 & 326 & 400 & +74 \\
\hline $2367 \cdot 0$ & 249 & 256 & +7 & 15352 & 154 & 272 & +118 \\
\hline 3516.6 & 314 & 312 & -2 & 18597 & 90 & 85 & -5 \\
\hline
\end{tabular}

J. Forged Ring Cast Steel (Hard).

Mean diameter . . $d=14 \cdot 25$ centim.

Bar-thickness . . $2 \mathrm{R}=1.421$,

Number of coils . . $n \cdot=1069$.

$\mu$ calc. $=45+90\left\{\sin \theta+\frac{1}{2} \cdot \sin 2 \theta\right\}$,

where

$$
\theta=\frac{\mathfrak{B}}{100} \text { degrees. }
$$

\begin{tabular}{|c|r|r|r||r|r|r|r|}
\hline \multirow{2}{*}{ 3. } & \multicolumn{2}{|c|}{$\mu}$. & \multirow{2}{*}{ Diffs. } & \multirow{3}{*}{ 3. } & \multicolumn{2}{|c|}{$\mu}$. & Diffs. \\
\cline { 2 - 7 } & Obs. & Calc. & & & Obs. & Calc. & \\
\hline 12.75 & 49 & 45 & -4 & 645 & 67 & 62 & -5 \\
$59 \cdot 48$ & 47 & 47 & 0 & 2189 & 115 & 110 & -5 \\
$120 \cdot 8$ & 48 & 48 & 0 & 4492 & 150 & 153 & -3 \\
$170 \cdot 4$ & 51 & 52 & +1 & 6496 & 157 & 161 & +4 \\
267.9 & 57 & 53 & -4 & 8959 & 134 & 135 & +1 \\
405 & 62 & 59 & -3 & 13870 & 70 & 60 & -10 \\
551 & 64 & 62 & -2 & & & & \\
\hline
\end{tabular}

First, as to the saturation-value. In nearly all the rings of crown iron the saturation-value of the induction is under 18,000 , although in ring $\mathrm{F}$ it appears to exceed 19,000. This agrees generally with Rowland's result as to rings.

In my paper on Electromagnets (Phil. Mag. xvii. p. 532) I have shown that in the case of the two bars there examined the saturation-values were higher, and did not appear to give evidence of having any fixed limit. This seems intelligible 
when we remember that in the bar the lines of force are crowded closely only at the equatorial section.

The formula by which Rowland represented his results was of the type

$$
\mu=\mathrm{A} \sin \left\{\frac{\mathbf{B}+a \mu+\mathrm{C}}{\mathrm{D}}\right\},
$$

in which $\mathrm{A}, \mathrm{C}, \mathrm{D}$, and $a$ are constants depending upon the kind and quality of the metal used. This type of formula is unmanageable; and it seemed to me necessary to attempt a more direct expression of the facts.

Rowland's formula offers the suggestion that we may regard values of $\mu$ which occur in these experiments as corresponding to the first half of a periodic change, the whole of which would be completed for a value of $\mathbf{B b}$ corresponding to about twice the saturation-value. This being so, it is theoretically possible, according to Fourier's theorem, to express any set of the experimental values of $\mu$ by a series of sines and cosines of an angle proportional to the induction and of the multiples of that angle; and the only difficulty is to find the coefficients of the different terms of the series.

Unfortunately the distribution of the values obtained does not admit of the application of the simplest form of harmonic analysis; so that the accurate determination of the coefficients is attended with a good deal of difficulty; and after trial of various methods of solution of equations, including an extended application of the method of least squares, it was found that a process of trial and error was capable of giving better results than could be obtained in any other way.

The representations thus obtained are not in all cases very close, but they are quite sufficient to show, by comparison of the different rings of the same iron, the uselessness of attempting to define minutely the properties of a given kind of iron. For the analysis of the different sets these are as valuable, on account of the clearness of the expressions, as the representations founded on my subsequent theory, which are as close or closer.

As to the size of the rings. There are five rings of crown iron, E, F, G, H, K. Of these, $\mathrm{E}, \mathrm{H}$ have approximately the same mean diameter; $F, G, K$ have mean diameters rather more than twice as great. $H, K$ have about the same barthickness, E, F bar-thickness nearly twice as great, and $\mathrm{G}$ a bar-thickness between three and four times as great. But when we examine the expressions for $\mu$, we fail to find any systematic differences which appear to correspond with these differences in the dimensions. So that, so far as this small number of experiments goes, the fundamental suppositions are 
justified in leading to values of $\mu$ independent of the dimensions of the rings.

It is a question of particular interest whether the iron in the interior of thick bars is in any degree shielded by that which encloses it from the effects of magnetic potential. In rings we have this question detached from the complications caused by the ends of straight bars or tubes, and are face to face with the simple question:-Is magnetic potential so ab. sorbed in its passage through external layers of iron that its effect in the interior is sensibly diminished?

To answer this question it is convenient to make use of the ordinary expression for magnetizing force $\left(=\frac{\mathbf{B}}{\mu}\right.$ in the above tables), and to tabulate the values of the induction in the different rings corresponding to' a series of definite values of the magnetizing-force. If, then, there is absorption of the force in the external layers, the inside of the thickest rings should be the most shielded, and the induction should throughout be lower in those rings for the same magnetizing force.

An inspection of the following Table will show that, if anything, the reverse of this is the case.

Table of Values of $\mathbf{Q B}$ in the different Crown Iron Rings, corresponding to certain Values of the Magnetizing Force.

\begin{tabular}{|c|c|c|c|c|c|}
\hline & $\mathrm{G}$ & $\mathrm{E}$ & $\mathrm{F}$ & $\mathrm{H}$ & $\mathbf{K}$ \\
\hline $\begin{array}{c}\text { Mean diam.... } \\
\text { Bar-thickness. }\end{array}$ & $\begin{array}{c}21 \cdot 5 \mathrm{~cm} . \\
2 \cdot 535\end{array}$ & $\begin{array}{c}10 \cdot 035 \mathrm{~cm} \\
1 \cdot 298\end{array}$ & $\begin{array}{c}22 \cdot 1 \mathrm{~cm} . \\
1 \cdot 292\end{array}$ & $\begin{array}{c}10 \cdot 735 \mathrm{~cm} . \\
\cdot 7137\end{array}$ & $\begin{array}{c}22 \cdot 725 \mathrm{~cm} . \\
7544\end{array}$ \\
\hline $\begin{array}{c}\text { Magnetizing } \\
\text { Force. }\end{array}$ & & & & & \\
$\cdot 2$ & 126 & & & & \\
$\cdot 5$ & 377 & 270 & 224 & $82 \cdot 4$ & 85 \\
1 & 1449 & 1293 & 840 & 675 & 214 \\
2 & 4564 & 3952 & 3533 & 2777 & 2417 \\
5 & 9900 & 9147 & 8293 & 8479 & 8884 \\
10 & 13023 & 13357 & 12540 & 11376 & 11388 \\
20 & 14911 & 14653 & 14710 & 14066 & 13273 \\
50 & 16217 & 15704 & 16062 & 15174 & 13890 \\
100 & 17148 & 16677 & 17900 & 16134 & 14837 \\
\hline
\end{tabular}

The thickest ring, $\mathrm{G}$, has the highest inductions throughout. The thinnest rings fall a little behind on the whole, except in the initial values. It seems probable that this may be due to slight flaws of small surface-extent, which may not perceptibly impair the magnetic conductivity of the thick ring, but may interfere with that of the thin ring. At all events the hypothesis of shielding is conclusively negatived.

Returning to the general tables, the variations which strike 
us most are those in the initial values of $\mu$ and those in the coefficient of $\sin \theta$. We can only conclude, either that the iron is of very variable quality, or that the forging of the rings is liable to introduce these large discrepancies.

I may mention here that, in accordance with. Rowland's direction, a coating of at least $\frac{1}{8}$ of an inch was removed in the lathe from the rings after forging. The ring $K$ was turned out of the very heart of a forged ring originally nearly four times its ultimate thickness.

The Lowmoor iron ring shows low initial permeability and a high saturation-point. In both respects it much resembles ring $\mathrm{F}$, but the maximum permeability of the Lowmoor is greater than that of $\mathrm{F}$, though considerably less than that of $\mathrm{E}$ or $\mathrm{G}$; in fact the differences due to the type of iron appear inconsiderable compared with the differences between different rings of the same iron.

The soft steel ring presents very remarkable results. First we notice that the saturation-point is decidedly high ; that is to say, by the employment of sufficient force, as shown by the low values of the permeability, the induction in the soft steel was actually raised to over 18,000 .

The initial permeability is about one third of the average value for soft iron; the maximum permeability about one fifth of the average value for soft iron. But the strangest thing of all is that the variable part of the permeability appears to be completely represented by a single term involving the sine of the angle proportional to the induction, so that the maximum permeability corresponds to an induction of about 9000 instead of to an induction of between 5000 and 6000 as is usual in soft iron.

In the case of the hard steel ring the power employed was insufficient to force the steel up to its saturation-point, and before the ring could be re-examined with a larger number of windings the tubes of the boiler broke down and put a stop to work for the present. But theobservations already obtained are very fairly represented by a formula which would give a high saturation-point. The initial permeability is less than one half that of the soft steel. The maximum permeability is about one third that of the soft steel, and it corresponds to a somewhat lower value of the induction than in soft iron, instead of to a higher value as in soft steel; that is to say, what we may call the octave term ( $\sin 2 \theta$ ), which vanishes for the soft steel, has for the hard steel the largest proportional value that occurs in any of the rings examined. The physical meaning of this will be given us by my new theory.

In Rowland's paper above referred to he observes that it is 
probable that Weber's theory may be so modified as to give an equation of the type he employs. I am not aware that this has been attempted, and it seems improbable on account of the nature of the equation. Further it is clear that the functions involved are continuous through the whole range of the magnetization. And the essence of Weber's theory and of all modifications of it is to divide the course of events into separate portions, in which different causes are supposed to operate, and different expressions are obtained for the results.

The statement that the results are continuous applies to the residual subpermanent magnetism as well as to that obtained by reversal; for although I have not made any determinations of residual magnetism, the data in Rowland's table I. are sufficient to give a series of values of residual magnetism which determine the point. Using the same angle proportional to the total induction for $\theta$, we can find a new quantity $\mu^{\prime}$, which we may call susceptibility to residual magnetism.

The following table gives the results of this process as applied to Rowland's table I.:-

$\mathbf{B B}$ or $Q$, total induction by reversal.

${\overrightarrow{2 B^{\prime}}}^{\prime}$ or $\mathrm{P}$, subpermanent or residual induction $(\mathrm{Q}-\mathrm{T})$.

$\mu$, Rowland's value of the permeability.

$$
\begin{gathered}
\text { Then, if } \mu^{\prime}=\frac{l \mathbf{B}^{\prime}}{\text { potential }} \text { in the same way that } \mu=\frac{l \mathbf{B}}{\text { potential }}, \\
\mu^{\prime}=\mu \frac{\mathbf{B}^{\prime}}{\overline{\mathbf{B}}},
\end{gathered}
$$

from which the following values are calculated:differences.

Initial values $\left\{\begin{array}{c}\mu_{0}=332 \\ \mu_{0}^{\prime}=69\end{array}\right\}$ from first and third lines, by

$\mu^{\prime}$ or Susceptibility to Subpermanent Magnetism, from

\begin{tabular}{|c|c|c|c|c|c|c|c|}
\hline $\begin{array}{c}\text { Z3. } \\
\text { Q. }\end{array}$ & $\begin{array}{c}\mathfrak{Z B}^{\prime} . \\
\text { P. }\end{array}$ & $\mu$. & $\mu^{\prime}$ & $\begin{array}{c}\text { B. } \\
\text { Q. }\end{array}$ & $\begin{array}{c}\mathbf{B}^{\prime} . \\
\text { P. }\end{array}$ & $\mu$. & $\mu^{\prime}$. \\
\hline 71.5 & $18 \cdot 7$ & $390 \cdot 7$ & 1027 & 8943 & 6369 & 2208 & 1572 \\
\hline 600.5 & $211 \cdot 1$ & $868 \cdot 7$ & $305 \cdot 4$ & 10080 & 6838 & 1899 & 1288 \\
\hline 9667 & $438 \cdot 7$ & $1129^{\circ}$ & $512 \cdot 4$ & 12270 & 7502 & 1448 & 885 \\
\hline 2460 & 1572 & 1936 & 1237 & 12970 & 7666 & 1269 & 750 \\
\hline 2923 & 1942 & 2078 & 1380 & 13630 & 7520 & 1137 & 627 \\
\hline 3082 & 2064 & 2124 & 1422 & 14540 & 7989 & $824 \cdot 1$ & 4528 \\
\hline 4959 & 3628 & 2433 & 1780 & 15770 & 8116 & 461.8 & $237 \cdot 6$ \\
\hline 5482 & 3811 & 2470 & 1717 & 16270 & 7852 & 353.8 & 1707 \\
\hline 5782 & $401]$ & 2472 & 1715 & 16600 & 7888 & 258 & 122.6 \\
\hline 6651 & 4746 & 2448 & 1747 & 17500 & & & \\
\hline 7473 & 5434 & 2367 & 1721 & & & & \\
\hline
\end{tabular}

Rowland's table I. 
There is a slight discontinuity here near the maximum value of $\mu^{\prime}$; but nothing to obscure the general applicability of a continuous formula.

We note that the initial value of $\mu^{\prime}$, though small, is by no means evanescent; that the curve formed by $\mu^{\prime}$ as ordinate and $\boldsymbol{B}$ as abscissa rises very steeply at starting; that $\mu^{\prime}$ has a maximum value equal to nearly two thirds of the maximum value of $\mu$; and that, although the position of this maximum of $\mu^{\prime}$ is somewhat obscured by the small discontinuity above referred to, yet it is substantially in the same position as the maximum of $\mu$.

On the whole, then, the facts are incompatible with those with which Weber's theory and Maxwell's modification of it are framed to correspond; and it will be of interest to consider whether there be any hypothesis by which these facts can be represented.

Prof. Hughes has recently put forward a theory, the fundamental supposition of which appears to be identical with that of the theory of Weber; but I cannot find that it has been applied so as to give any quantitative account of magnetizing functions or permeabilities.

I will now give an illustration of the suppositions as to molecular magnets, which would have to be made, in order that the principal term of the permeability may be proportional to the sine of an angle whose zero corresponds approximately to the zero of magnetic induction, and $180^{\circ}$ to saturation, the angle being proportional to magnetic induction in between. The resulting theory is of an impossible character.

Let a typical molecule be represented by a small inagnet hung by a torsion-wire at the centre of a coil, and let the magnet be placed so that when no current is passing it stands nearly at right angles to the plane of the coil, but with the poles in the opposite direction to that which the action of the coil tends to make them assume. Suppose, then, that a current is sent through the coil; the needle will be deflected, and will rest at the point where the deflecting couple on the needle is balanced by the torsion of the wire. Now the couple due to the coil may be expressed as $\mathrm{GC} \sin \theta$; where $\mathrm{C}$ is the current, $\mathrm{G}$ a constant, and $\theta$ the deflection from a position at right angles to the coil. The torsion may be represented by $\tau(\theta-\alpha)$, where $\alpha$ is small; or approximately by $\tau \theta$, whence we have for the position of rest

$$
\tau \theta=\mathrm{GC} \sin \theta .
$$


Let $\theta$, or the rotation, represent the magnetic induction. It is clear that, however great $\mathrm{C}$ may be, it can never tend to increase $\theta$ beyond $180^{\circ}$, since this is the position in which the coil tends to place the needle independently of the torsion: this represents saturation. Further, $\mathrm{C}$ is proportional to the magnetic potential or magnetizing force, so that $\frac{\theta}{\overline{\mathrm{C}}}$ is proportional to $\mu$ and $\frac{\theta}{\mathrm{C}}=\frac{\mathrm{G}}{\tau} \sin \theta$; so that $\mu$ is represented approximately by a quantity proportional to the sine of an angle proportional to the induction.

If the fundamental ideas of this illustration could be in any way applied to the behaviour of systems of molecules, it would be easy, by small modifications of the principal suppositions, to take count of the smaller terms; but it seems quite impossible to carry out this application; and the illustration is only useful as showing the sort of circumstances which would have to be imagined in order to obtain a formula of this sort from the hypothesis of molecular magnets.

I will proceed to sketch another theory which appears to me more reasonable, and leads to forms of function by which we can represent the experiments very well.

Suppose that every molecule of iron has one axis, and only one axis, through which magnetism can be transmitted.

The molecules in an inert mass of iron are supposed to have their axes of transmission distributed in all directions. There will then be a very small proportion of molecules whose axes are so situated as to form continuous lines of transmissibility for magnetic lines of force proceeding in a given direction. Thus, for small magnetizing forces there will be a small transmissibility or permeability.

In the case in which molecules are so situated that the extremities of their axes do not join, the magnetic lines of force have to pass between the molecules as they would through ordinary space. We suppose that the transmissibility through the axes of the molecules is very great compared with that in ordinary space or in the interstices of the molecules.

As the magnetic induction increases, couples are established by the action of the lines of force, which tend to move the axes of the molecules towards the direction of the lines of force.

Consider a single molecule. Let the plane of the paper cut it in the plane containing its magnetic transmission axis and the axis of magnetization. Then the lines of force will traverse the molecule somewhat in the way shown in the 

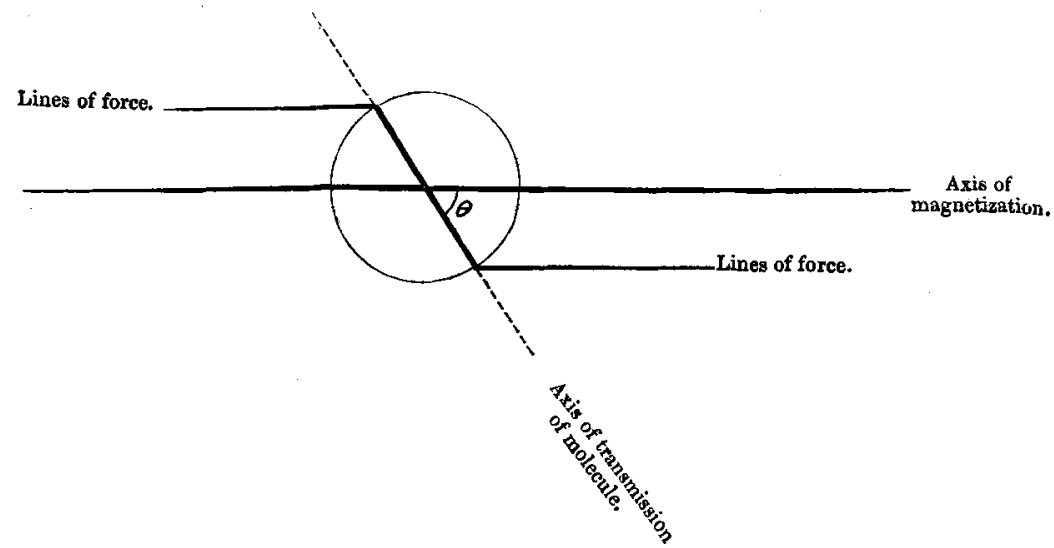

above figure, though they will only be parallel on the average to the mean axis of magnetization, where they issue from the molecule.

Now it is known that many magnetic phenomena may be expressed by the hypothesis of a tension along the lines of force. If we suppose such a tension to exist here, we have a couple whose force is equal to the tension, and arm equal to the diameter of the molecule $\times \sin \theta$, tending to turn the axis of the molecule towards the axis of magnetization. If we suppose the molecules to give way to this force, the ends of the axes of the successive molecules approach each other, and the intermediate space, as well as the number of molecules, required to be traversed is diminished. This accounts for the increase of the permeability in the first part of the range of the induction.

Without attempting to frame exact hypotheses we ean see generally that the whole magnetic resistance may be expressed by supposing the lines of force to form a zigzag, the obliquity of which diminishes as the tension of the lines of force increases. From this representation, assuming a law similar to Ohm's law for electric circuits, we get the expression $\frac{l}{\cos \delta}$ as representing the reduced resistance of a length $l$ of the magnetic substance, $\delta$ being an auxiliary angle representing the mean obliquity of the zigzag. We have further to take into account the fact that all forms of iron and steel are capable of transmitting. only a limited amount of magnetic induction throngh a given area. In the absence of any accurate knowledge as to the law which governs this part of the resistance of the molecules to the lines of force, I shall 
assume a factor in the permeability which vanishes for the saturation-value, namely $\left(\mathbf{B B}_{\infty}-\mathbf{Q B}\right)$, where $\mathbf{B B}_{\infty}$ is the saturationvalue of the induction. The resistance will then be proportional to the reduced length divided by this factor, so that resistance

$$
\begin{aligned}
& =\frac{l}{\mu} \\
& =\frac{l}{\mathrm{~A}\left(\mathbf{a B}_{\infty}-\mathbf{a} \mathbf{B}\right) \cos \delta}, \\
\mu & =\mathrm{A}\left(\mathbf{B}_{\infty}-\mathbf{B}\right) \cos \delta .
\end{aligned}
$$

or

We may give some further account of the factor $\left(\mathbf{B}_{\infty}-\mathbf{B B}\right)$ based on an analogy between the lines of magnetic force and solid wires. If a number of thin wires had to be packed into a cylindrical tube, by the time there were a certain number of them the hollow of the tube would be full, and no more could be got in. Also, if there were a less number, the space available in the tube would be measured by the number of wires that could still be added.

Now if our axis of transmission in the molecule represent the hollow tube, and the lines of force the wires, it is reasonable to assume that the permeability is proportional to the portion of the channel left open and unoccupied. And this is precisely given by $\left(\mathbf{B B}_{\infty}-\mathbf{B}\right)$. It is only an analogy, but it seems to me not destitute of force. We may call $\left(\mathbf{B}_{\infty}-\mathbf{B B}\right)$ "defect of saturation."

Let us return to the consideration of the equilibrium of the molecule. On the one hand we have the couple exerted by the lines of force; on the other that arising from molecular attachments, which tends in the opposite direction.

The simplest law of molecular attachments is the law of torsion; and in considering a single molecule $I$ assume that the couple tending to restore it to its place is proportional to the angular rotation from the position of rest. (The displacements of subpermanent and permanent magnetism are at first left out of the question.) If therefore

$\rho$ be the diameter of the molecule,

$k$ the torsion per degree,

$\phi$ displacement from rest,

$\theta$ inclination to direction of magnetization in disturbed position,

$$
\text { BB* } \rho \sin \theta=k \omega \phi
$$

* I assume that the tension along the lines of force is proportional to $\mathbf{B}$ instead of to $\mathbf{B}_{2}$ according to Maxwell. I shall return to the point; but may say here that the assumption of force proportional to $\mathbf{Z B}_{2}$ led to formulæ which failed to represent the experiments. 
expresses the condition of equilibrium; $\omega$ is the unit angle, so that $\omega \phi$ is $\phi$ expressed in degrees.

If we pass from the consideration of a single molecule to that of a molecular arrangement we are confronted with problems which, for the most part, must be regarded as insoluble. One point, however, is clear.

If the axes of the molecules are arranged uniformly in all directions, the average inclination to any one line is $60^{\circ}$.

For we may suppose the axes of transmissibility uniformly distributed over the surface of each hemisphere, and the hemisphere is divided into two equal portions by a cone whose semi-vertical angle is $60^{\circ}$.

If therefore we represent the condition of the average molecule with respect to inclination by a single molecule, we must assume the initial inclination to the axis of magnetization to be $60^{\circ}$.

And, in the above expression, when $\theta=0, \omega \phi=60^{\circ}$; so that $\omega \phi=60^{\circ}-\omega \theta$. And the equation becomes,

$$
\mathbf{B}=\frac{k}{\rho} \frac{60^{\circ}-\omega \theta}{\sin \theta} \text {. }
$$

It only remains to connect $\theta$ with the auxiliary angle $\delta$. In the absence of exact knowledge of the molecular arrangements, the only thing we can do is to assume the simplest connection possible. We shall assume

$$
\delta=f \theta,
$$

where $f$ is a factor. We thus obtain the system of equations,

$$
\begin{aligned}
\mu & =\mathrm{A}\left(\mathbf{B}_{\infty}-\mathbf{B}\right) \cos \delta, \\
\delta & =f \theta, \\
\mathfrak{B} & =\frac{k}{\rho} \frac{60^{\circ}-\omega \theta}{\sin \theta} .
\end{aligned}
$$

This system of equations involves only the arbitrary constants $\mathrm{A}, \mathbf{\mathbf { B }}_{\infty}, f$, and $\frac{k}{\rho}$, for $60^{\circ}$ cannot be called arbitrary. The equations are capable of representing very well the connection between $\mu$ and $\mathbf{\Re}$. The Fourier's series are in some respects more flexible. Bat these equations are important as regards their physical bearing. As the computations present some difficulty, I here give a systematic scheme for adapting these equations to the representation of experimental series of values of $\mathbf{B}$ and $\mu$.

Given a series of experimental values of $\mathbf{B}$ and $\mu$, it is required to represent them by the formulæ 


$$
\begin{aligned}
& \mu=\mathrm{A}\left(\mathbf{B}_{\infty}-\mathbf{B}\right) \cos \delta, \text {. . . . . } \\
& \delta=f \theta, \text {. . . . . . . . . . } \\
& \mathbf{A B}=\frac{k}{\rho} \frac{60^{\circ}-\theta \omega}{\sin \theta} . \quad . \quad . \quad . \quad . \quad .
\end{aligned}
$$

Let $\mathbf{B}_{1}, \mu_{1}$ be the lowest pair of values given ;

$\mathbf{B}_{2}, \mu_{2}$ those corresponding to $\mu_{2}$, the maximum value of $\mu$; $\mathbf{B}_{\infty}$ the saturation-value of $\mathbf{1 3}$ as estimated from the experiments or assumed.

The first process aims at determining $A$ by trial and error, under the condition that the maximum value of $\mu$ represented by the formula shall belong to the value $\mathbf{B}_{2}$ given by experiment. The theory of this is as follows.

Since $\mu$ is a maximum, we have from (1),

$$
d \mu=0=-d \mathbf{B} \cos \delta-\left(\mathbf{B}_{\infty}-\mathbf{B}\right) \sin \delta d \delta,
$$

or

whence from (2),

$$
\tan \delta=-\frac{\frac{d \mathfrak{B}}{d \delta}}{\left(\mathbf{B B}_{\infty}-\mathbf{B B}\right)} ;
$$

Again, differentiating (3),

$$
\tan \delta=-\frac{1}{f} \frac{\frac{d \mathfrak{B}}{d \theta}}{\left(\mathbf{B B}_{\infty}-\mathbf{1 B}\right)}
$$

$$
d \mathbf{B} \sin \theta+\mathbf{B} \cos \theta d \theta=-\frac{\boldsymbol{\omega}}{\rho} d \theta
$$

or

$$
\frac{d \mathfrak{B}}{d \theta}=-\frac{\mathfrak{B}+\frac{\omega k}{\rho} \sec \theta}{\tan \theta} . .
$$

Combining (4) and (5),

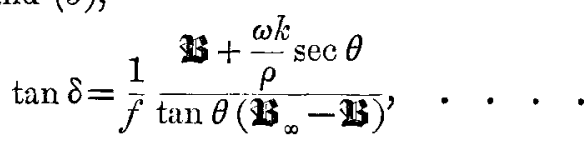

which is the condition for the maximum value of $\mu$.

The work proceeds as follows:-

Assume a value of $A,\left(A^{\prime}\right)$.

Find $\omega \delta_{1}$ from $\cos \delta_{1}=\frac{\mu_{1}}{\Lambda^{\prime}\left(\mathbf{b}_{\infty}-\mathbf{b}_{1}\right)}$,

$$
\omega \delta_{2} \text { from } \cos \delta_{2}=\frac{\mu_{2}}{\bar{A}^{\prime}\left(\mathbf{b}_{\propto}-\mathbf{B b}_{2}\right)}
$$

Phil. Mag. S. 5. Vol. 19. No. 117. Fel. 1885. 
$(\omega \times$ angle is used throughout to represent degrees, $\omega$ being

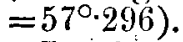

Let first approximation be denoted by one accent, second by two, and so on.

Assume $\omega \theta_{1}^{\prime}=60^{\circ}$. Then

$$
f^{\prime}=\frac{\omega \delta_{1}}{60^{\circ}}
$$

whence

$$
\begin{aligned}
\omega \theta_{2}^{\prime} & =\frac{\omega \delta_{2}}{f^{\prime}} . \\
\frac{k^{\prime}}{\rho} & =\frac{\mathfrak{B}_{2} \sin \theta_{2}{ }^{\prime}}{60^{\circ}-\omega \theta_{2}^{\prime}} .
\end{aligned}
$$

Second approximation.

$$
\begin{aligned}
\omega \theta_{1}^{\prime \prime} & =60^{\circ}-\frac{\sin \theta_{1}{ }^{\mathbf{B B}_{1}}}{\frac{k^{\prime}}{\rho}}, \\
f^{\prime \prime} & =\frac{\omega \delta_{1}}{\omega \theta_{1}{ }^{\prime \prime}} ;
\end{aligned}
$$

whence

$$
\begin{aligned}
\omega \theta_{2}^{\prime \prime} & =\frac{\omega \delta_{2}}{f^{\prime \prime}}, \\
\frac{k^{\prime \prime}}{\rho} & =\frac{\mathbf{B}_{2} \sin \theta_{2}^{\prime \prime}}{60^{\circ}-\omega \theta_{2}^{\prime \prime}} .
\end{aligned}
$$

In general this will be enough, but there is no difficulty in repeating the process as often as necessary.

Dropping the accents, we have for calculating the righthand side of (6),

$$
\mathbf{a}_{2}, \mu_{2}, \omega \theta_{2}, f, \frac{k}{\rho}
$$

From the result find $\tan \delta$, whence $\delta$, or rather $\omega \delta$.

If the condition as to the maximum is satisfied by the assumed value of $A$, this $\omega \delta$ will coincide with $\omega \delta_{2}$. If they differ, as they generally will, the assumed value of $\mathrm{A}$ is wrong. If $\omega\left(\delta-\delta_{2}\right)^{\prime}$ is positive, $A$ has to be increased, and vice versa

Another hypothesis as to the value of $\mathrm{A}$ has then to be made, and the work repeated, $\left(\mathrm{A}^{\prime \prime}\right)$.

The final value $\omega\left(\theta-\theta_{2}\right)^{\prime \prime}$ thus obtained, compared with $\omega\left(\theta-\theta_{2}\right)^{\prime}$, offers a means of estimating the true value of $A$, for which the maximum of $\mu$ given by the equations corresponds to the proper value of $\mathbf{B}$. But the convergence of the process is not quick enough to enable differences to be used for the estimation, unless close approximation l as been already 
attained. In this case $\mathrm{A}$ may be estimated from the formula

$$
\mathrm{A}^{\prime}-\left(\mathrm{A}^{\prime}-\mathrm{A}^{\prime \prime}\right) \frac{\omega\left(\delta-\delta_{2}\right)^{\prime}}{\omega\left(\delta-\delta_{2}\right)^{\prime}-\omega\left(\delta-\delta_{2}\right)^{\prime \prime}},
$$

where one accent refers to the first of two assumptions, two to the second.

Before this way of estimating $A$ was devised, the only way of approximating to it was to carry out the whole calculation of $\mu$ from $\mathbf{k}$ for each assumed value of $\mathrm{A}$, and then judge from the results. It was soon seen that the representations obtained differed in the position of the maximum of $\mu$. The following correspondences were thus obtained for ring E:-

Assumed.

A.

1 . . Between 7000 and 11,000.

$\frac{1}{2}$. . . About 7000 , still too late.

$\frac{1}{3}$. . . Before 6400 , a little too early.

An accurate judgment could be formed by the correspondences of $\mu$ before and behind the maximum.

The application of the above method saved the great labour of these complete computations by way of trial.

It is necessury to repeat the above processes until the resulting value of $\omega\left(\delta-\delta_{2}\right)$ is sensibly zero. The quantities $f$ and $\frac{k}{\rho}$ obtained in the computation which satisfies this condition are taken on for the final process.

The next thing is to form the table connecting $\mathbf{3}$ and $\theta$, from equation (3). This is most conveniently done by forming a table of $\log \frac{60^{\circ}-\omega \theta}{\sin \theta}$ once for all, for each degree from about $20^{\circ}$ to $60^{\circ *}$. The addition of these logarithms to that of $\frac{k}{\rho}$ gives the values of $\mathbf{3}$ corresponding to the degrees of $\omega \theta$.

This table $(\boldsymbol{B}, \theta)$ is written out, with differences. The values of $\omega \theta$ corresponding to the experimental values of $\mathbf{2 B}$ are then found by interpolation.

Multiplying $\omega \theta$ by $f$, we find $\omega \delta$ corresponding to each experimental value of $\mathbf{B}$.

With $\mathbf{A}, \omega \delta$, and $\mathbf{B}, \mu$ is calculated from equation (1) for each experimental number $\mathbf{B}$.

The following are comparisons of this theory with experiment.

* For steel the table is formed for every $10^{\prime \prime}$ from $60^{\circ}$ to $59^{\circ} 59^{\prime}$, and then for every minute down to $59^{\circ}$. 
Equations.

$$
\begin{aligned}
\mu=\mathrm{A}\left(\mathbf{B}_{\infty}-\mathbf{B}\right) & \cos \delta, \quad \delta=f \theta, \quad \mathbf{B}=\frac{k}{\rho} \frac{60^{\circ}-\omega \theta}{\sin \theta} . \\
\text { Ring E. } \quad \mu & =\cdot 3856(17,500-\mathbf{B}) \cos \delta, \\
\log \frac{k}{\rho} & =2 \cdot 32208, \quad \log f=\cdot 16153 .
\end{aligned}
$$

\begin{tabular}{|c|c|c|c|c|c|}
\hline \multirow{2}{*}{ BB. } & \multirow{2}{*}{$\theta$. } & \multirow{2}{*}{$\delta}$. & \multicolumn{2}{|c|}{$\mu$} & \multirow{2}{*}{ Diffs. } \\
\hline & & & Calc. & Obs. & \\
\hline 1297 & $5 \hat{9} 28$ & $8 \stackrel{\circ}{86}$ & 436 & 437 & -1 \\
\hline $239 \cdot 1$ & 592 & 864 & 457 & 493 & -36 \\
\hline $2174 \cdot 3$ & 5151 & $\begin{array}{lll}75 & 13\end{array}$ & 1508 & 1474 & +34 \\
\hline 3187 & $48 \quad 36$ & 7030 & 1842 & 1817 & +25 \\
\hline 5182 & 437 & 6233 & 2190 & 2163 & +27 \\
\hline 6403 & 4017 & $58 \quad 26$ & 2235 & 2234 & +1 \\
\hline 7208 & 3837 & $\begin{array}{lll}56 & 10\end{array}$ & 2210 & 2206 & +4 \\
\hline 11516 & 3125 & 4534 & 1615 & 1615 & 0 \\
\hline 13276 & 2911 & $42 \quad 20$ & 1204 & 1382 & -178 \\
\hline 14017 & 2820 & 416 & 1012 & 1047 & -35 \\
\hline 15000 & 2716 & $40 \quad 8$ & 737 & 635 & +102 \\
\hline 16380 & $\begin{array}{l}25 \quad 55 \\
5\end{array}$ & 3725 & 342 & 218 & +124 \\
\hline 17245 & 2453 & $\begin{array}{ll}36 & 6\end{array}$ & 79 & 117 & -38 \\
\hline
\end{tabular}

\begin{tabular}{|c|c|c|c|c|c|}
\hline \multirow{2}{*}{$\mathfrak{Z}$. } & \multirow{2}{*}{$\theta$. } & \multirow{2}{*}{$\delta$} & \multicolumn{2}{|c|}{$\mu$. } & \multirow{2}{*}{ Diff, } \\
\hline & & & Calc. & Obs. & \\
\hline $57 \cdot 6$ & 5959667 & $8 \hat{9} 58 \quad \tilde{5}$ & 122.3 & 123 & \\
\hline 277 & $\begin{array}{llll}59 & 59 & 45 \%\end{array}$ & $\begin{array}{lll}89 & 57 & 50\end{array}$ & 137 & 156 & -19 \\
\hline 1826 & $59 \quad 58 \quad 28$ & $8955 \quad 52$ & 240 & 248 & -8 \\
\hline 2367 & $5958 \quad 00$ & $\begin{array}{lll}89 & 55 & 16\end{array}$ & 267 & 249 & +18 \\
\hline 3517 & $\begin{array}{lll}59 & 57 & 2\end{array}$ & 895346 & 329 & 314 & +15 \\
\hline 5816 & $5955 \quad 6$ & $8950 \quad 53$ & 414 & 420 & -6 \\
\hline 9468 & $\begin{array}{lll}59 & 52 \quad 2\end{array}$ & $\begin{array}{llll}89 & 46 & 19\end{array}$ & 461 & 461 & 0 \\
\hline 11497 & $\begin{array}{lll}59 & 50 & 19\end{array}$ & $8943 \quad 41$ & 444 & 443 & +1 \\
\hline 12443 & 594931 & $8942 \quad 29$ & 424 & 326 & +102 \\
\hline 15352 & $5947 \quad 5$ & $8938 \quad 53$ & 314 & 154 & +160 \\
\hline 18597 & 594420 & 893441 & 114 & 90 & +24 \\
\hline
\end{tabular}

J. Soft Steel. $\quad \mu=11(20,000-\mathbf{B}) \cos \delta$, $\log \frac{k}{\rho}=4 \cdot 78936, \quad \log f=\cdot 17595$.

Here $\mathbf{B B}_{\infty}$ is a little overestimated. Probably 19,500 would be nearer the mark.

$A$ is the permeability of the molecules themselves per unit defect of saturation. For, putting $\theta=\delta=0$, or supposing the 
axes all arranged in continuous chains parallel to the axis of magnetization, then

$$
A=\frac{\mu}{2 B_{\propto}-2 B} .
$$

The molecular permeability for given $\mathbf{B}$, under these circumstances, is therefore

$$
\mu=\mathrm{A}\left(\mathbf{b}_{\propto}-\mathbf{B}\right),
$$

which increases continually as $\mathbf{B}$ diminishes, and has the maximum values $(\mathbf{B}=0)$,

$$
\begin{aligned}
& \text { Ring } \mathrm{E} \text {. . . } \mu=6748 \text {, } \\
& J \text { (soft steel) } \quad \mu=220,000 \text {. }
\end{aligned}
$$

Nothing is more surprising in these results than the enormous values of the molecular permeability of the soft steel, and the way in which the effect of this is suppressed by the comparative immobility of the molecules. Thus:-

$$
\begin{gathered}
\text { Extreme variation } \\
\text { of average } \\
\text { inclination: }
\end{gathered}
$$

Extreme rotation of average molecule.

Soft iron ring $\mathrm{E} \quad .60^{\circ} \ldots 24^{\circ} 53^{\prime}$ $33^{\circ} 7^{\prime}$

Soft steel J . . . $60^{\circ} \ldots 59^{\circ} 44^{\prime} 20^{\prime \prime} 15^{\prime} 40^{\prime \prime}$

The molecular permeability of the soft steel is 28.5 times as great as that of the soft iron. The extreme rotation of the average molecule is 127 times as great in the soft iron as in the soft steel.

$$
\frac{k}{\rho} \text { is } 287 \text { times as great in the soft steel as in the soft iron; }
$$

so that, assuming that the mean diameter of the molecules is the same, the force of torsion arising from molecular attachment has this ratio in the two cases.

Putting together the conditions associated with early and late maxima in the Fourier's-series expressions and those of the present theory, we have

$$
\text { Early Maxima. }
$$

Late Maxima.

Fourier's series. $\left\{\begin{array}{c}\text { Octave and higher Octave and higher } \\ \text { terms well developed. torms small or absent. }\end{array}\right.$ $\left.\begin{array}{c}\text { New magnetic } \\ \text { equations. }\end{array}\right\}$. A small.

A large.

Recalling the meaning of $A$, we see that the development of the octave and higher terms in the Fourier's series corresponds to low molecular permeability, other things being equal*. It

* A depends on the absolute magnitudes of the $\mu$ 's to be represented as well as on their course. 


\section{Professors Reinold and Rückor on the Influence of an}

will be of interest to see, when the hard-steel experiments are complete, how far the resulting inference of low molecular permeability in that case is borne out.

As to the question whether the tension of the lines of force is proportional to $\mathbf{B}$ or to $\mathbf{B}_{2}$, it is a question for experiment, and I shall take an early opportunity of endeavouring to decide it. But in the mean time it appears quite clear that if lines of force mean anything, it is that their number is proportional to the force. Consider a magnet-pole. We may represent the distribution of force about it either by the law of the inverse square of the distance, or by a system of lines of force radiating from the pole. If the two are equiralent, it involves the consequence that the force at any point is proportional to the number of lines of force.

XII. The Influence of an Electric Current in Modifying the Rate of Thinning of a Liquid Film. By Prof. A. W. RenNoLd, F.R.S., and Prof. A. W. Rücker, F.R.S.**

$\mathrm{I}^{\mathrm{N}}$ 1877 the results of some experiments made by us on liquid films were published in the Proceedings of the Royal Society (Proc. Roy. Soc., No. 182, 1877). One object of the investigation was the determination of the electrical resistance of the films. The method of Wheatstone's Bridge was employed for this purpose, and the current was passed through a film only at the moment when an observation was required. Under these circumstances the films generally thinned until they became black; and we succeeded in obtaining several measures of their resistance when this colour was displayed. Subsequently this method was abandoned for another, in which continuous currents were passed through the film, and the difference of potential between two fine wires thrust into it was measured by an electrometer, and compared with that between two other points in the same circuit separated by a known resistancet.

Although in many respects a great improvement, this method was in one point inferior to that previously employed. The behaviour of the films was very irregular when compared with that of those previously examined by the galvanometer, although they were formed of a liquid having the same composition. Sometimes they thinned rapidly, sometimes slowly;

* Communicated by the Physical Society. Read December 13, 1884. $\uparrow$ "On the Electrical Resistance of Thin Liquid Films, with a Revision of Newton's Table of Colours," Phil. Trans. 1881. 